\title{
The Effects of Sustained Release Metofluthrin on the Biting, Movement, and Mortality of Aedes aegypti in a Domestic Setting
}

\author{
Luke P. Rapley, Richard C. Russell, Brian L. Montgomery, and Scott A. Ritchie* \\ School of Public Health, Tropical Medicine and Rehabilitation Sciences, James Cook University, Cairns, Queensland, Australia; \\ Department of Medical Entomology, University of Sydney, Westmead Hospital, Westmead, NSW, Australia; \\ Tropical Population Health Services, Queensland Health, Cairns, Queensland, Australia; School of Public Health, \\ Tropical Medicine and Rehabilitation Sciences, James Cook University, Cairns, Australia
}

\begin{abstract}
The impact of a sustained release metofluthrin emanator and an allethrin-based mosquito coil on biting, movement and mortality of female Aedes aegypti was assessed in an apartment. In the room in which the metofluthrin emanator was activated, mosquito biting counts were reduced to zero. Metofluthrin also had a spillover effect, significantly $(P<0.001)$ reducing biting counts in a neighboring room 1,4 , and 24 hours after the emanator was activated when compared with either the coil or control (untreated) treatment. Mosquitoes were neither repelled nor expelled from a room exposed to metofluthrin. Indeed, a significantly $(P=0.023)$ greater proportion of mosquitoes were found in the treated room after exposure to metofluthrin when compared with either the coil or control treatment. Furthermore, in the room treated with metofluthrin the majority of mosquitoes died and a spillover effect into the neighboring room caused greater than one-third mortality of the mosquitoes. Metofluthrin could be used to prevent dengue transmission within a household.
\end{abstract}

\section{INTRODUCTION}

In northeastern Australia and throughout the tropical regions of the world the yellow fever mosquito, Aedes aegypti (L.) is a primary vector of dengue viruses. ${ }^{1,2}$ This day-biting mosquito has a close association with the urban environment, ${ }^{3,4}$ almost exclusively feeding on human hosts ${ }^{5,6}$ and breeding in artificial containers found in the domestic setting (i.e., pot plant bases, tires, roof gutters, etc.). ${ }^{7}$ Aedes aegypti regularly take multiple blood meals, often from different humans, to complete their gonotrophic cycle. ${ }^{6}$ Multiple blood feeding is epidemiologically important because it increases the probability of a mosquito contracting or transmitting a virus infection, often leading to clusters of dengue virus infections to occur in time and space (i.e., family and neighboring units). ${ }^{5}$

It is therefore imperative that during a dengue outbreak people infected with the virus and their closest neighbors take precautions to prevent vectors biting to interrupt the virus transmission cycle. Traditionally, mosquito coils and insecticidal vaporizing mats or liquids have been used to deter mosquitoes from biting, but these products are limited in their use by the requirement for a heat or power source to vaporize their active ingredient, commonly a synthetic pyrethroid such as d-allethrin or prallethrin. ${ }^{8-10}$ Furthermore, burning mosquito coils release significant amounts of fine particulate matter and volatile organic compounds that may pose an acute and chronic risk to human health. ${ }^{11}$

However, studies into the insecticidal activity of norchrysanthemic acid esters with high vapor activity at ambient temperature by Ujiahra and others ${ }^{12}$ identified a synthetic pyrethroid insecticide ("metofluthrin") that doesn't require a heating source and was extremely effective at knocking down Culex pipiens pallens in the laboratory. Metofluthrin has also been shown, with aspirator collections, to provide spatial repellency to mosquitoes in outdoor shelters without walls ${ }^{13,14}$

*Address correspondence to Scott A. Ritchie, School of Public Health, Tropical Medicine and Rehabilitation Sciences, James Cook University, PO Box 6811, Cairns, QLD 4870, Australia. E-mails: Scott.Ritchie@jcu.edu.au or scott_ritchie@health.qld.gov.au and in houses. ${ }^{15-17}$ Furthermore, metofluthrin has significantly reduced landing rates of Aedes species, ${ }^{8,9}$ and reduced the number of Aedes albopictus caught in Centers for Disease Control and Prevention (CDC)-type miniature mosquito traps ${ }^{18}$ in field trials. However, these studies failed to elucidate mosquito movement associated with metofluthrin exposure or the fate of mosquitoes after exposure. To achieve this we exposed marked cohorts of female Ae. aegypti mosquitoes to metofluthrin in an indoor domestic environment to measure the effects of its impact on 1) mosquito-human biting, 2) mosquito repulsion/expulsion, and 3) mosquito mortality.

\section{MATERIALS AND METHODS}

Experimental rooms. Our research was conducted in a two bedroom apartment in Cairns, Queensland, Australia in June and July 2007. The two adjacent rooms used for the experiment were of similar size (Room 1: $3.8 \times 2.6 \times 2.45 \mathrm{~m}=24.2 \mathrm{~m}^{3}$; Room 2: $\left.3.8 \times 2.75 \times 2.45 \mathrm{~m}=25.6 \mathrm{~m}^{3}\right)$ and were connected by a $2.0 \times 0.8 \mathrm{~m}$ door. Mosquitoes were contained within the experimental rooms by sealing doorways to the adjoining living room and bathroom with white cotton curtain using duct tape. A zipper in the living room curtain allowed us to enter the treatment rooms. The door between the bedrooms was left open to allow mosquitoes free access to both rooms. The floor of each experimental room was covered with white drop sheets to facilitate collection of dead mosquitoes at the end of each treatment replicate (after 24 hours). Each experimental room had a wardrobe containing clothing and was furnished with a black suitcase, green box, black laundry hamper, black pot, and a cardboard box that could be used by mosquitoes for harborage (Figure 1). Windows in each room were left open but were screened to prevent mosquito escape, and an overhead fan set on "low" to provide ventilation. By using insecticidal treatments in only one room at a time, and by releasing a uniquely marked cohort of mosquitoes in each room, we could determine if mosquitoes traveled freely between rooms, and the mortality of each cohort in each room.

Treatments and replication. The metofluthrin emanator was a $20 \times 15 \times 10 \mathrm{~cm}$ lightweight and portable concertina tower 


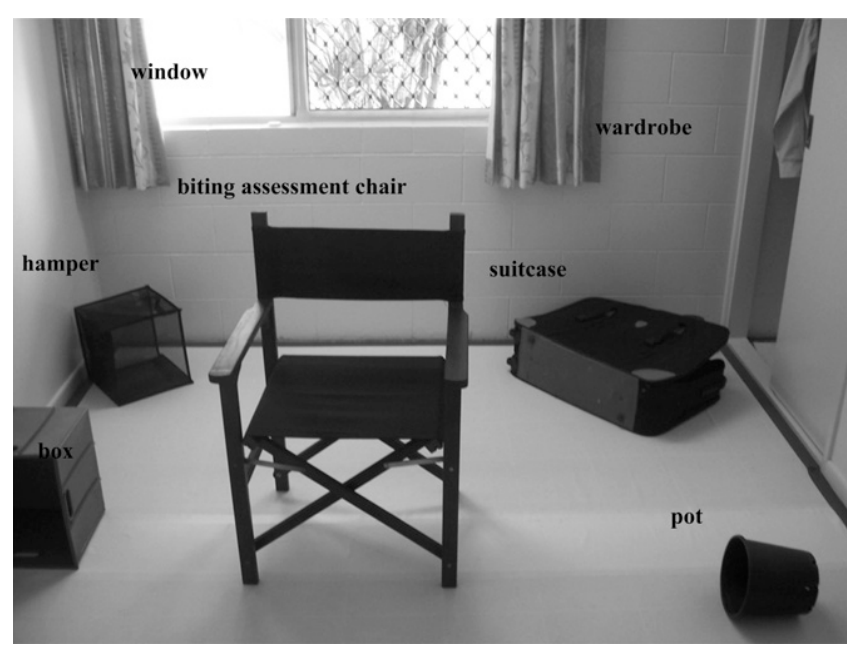

FIGURE 1. Experimental room with a chair used for mosquito biting assessments (center) and mosquito harborage sites (cardboard box is not in figure).

of metofluthrin-impregnated paper (containing $26 \mathrm{mg} \mathrm{AI}$ ) (Reckitt Benckiser Pty. Ltd., West Ryde, New South Wales Australia) that passively dispensed metofluthrin for $\sim 80$ hours at $28^{\circ} \mathrm{C}$ (Graeme Smith, Reckitt Benckiser Pty Ltd., unpublished data). The small, lightweight towers are portable and easily moved to provide personal protection from mosquitoes biting. The effect the metofluthrin-impregnated tower had on Ae. aegypti biting, movement, and mortality in a room was compared with that elicited by a standard Mortein coil $(0.2 \%$ d-allethrin; Reckitt Benckiser Pty. Ltd.) in the room, and a control (with neither tower nor coil in the room). Uniquely marked cohorts of 20 female Ae. aegypti were exposed to each pesticide treatment within the treatment room while the adjacent room was untreated. One replicate of each treatment was conducted in succession (i.e., metofluthrin, coil, and then control) and the order in which they were performed was randomized; there were six replicates in total. To minimize room effect on mosquito behavior, the treated room was alternated with the neighboring room after three replicates. New metofluthrin strips and coils were used for each trial and run for 24 hours, although the coil only had a lifespan of 8 hours. After each metofluthrin and coil trial, the apartment was aired out for 48 and 24 hours, respectively, to dissipate residual pyrethroid insecticide.

Mosquito rearing, sexing, and dusting. Mosquitoes used were laboratory reared, third generation Ae. aegypti originally collected from Parramatta Park, a suburb of Cairns. These mosquitoes were reared in a partially controlled temperature (range $20-29^{\circ} \mathrm{C}$ ) environment. Eggs were hatched in hay infusion and larvae were reared in metal trays on a cat food (Purina Friskies dry adult cat food, Nestle Purina Petcare, North Ryde, New South Wales, Australia) diet until pupation. Adult Ae. aegypti were kept in $35 \times 25 \times 15$-cm plastic cages and offered a $5-10 \%$ honey solution. To sort mosquitoes, the cage was placed in a $5^{\circ} \mathrm{C}$ cool room for 5 minutes to stupefy the mosquitoes. Females were then removed using a mouth aspirator and cohorts of 20,4-11-day-old, non-bloodfed female Ae. aegypti were placed into 1-L plastic holding containers. These containers were then held at room temperature until mosquitoes became active ( $\sim 30$ minutes) at which time they were dusted with Radglo fluoro paint using an insufflator. ${ }^{19}$ Two sets of two colors were used to mark treatment and control cohorts (set one: blue and magenta; set two: yellow and orange), and were alternated with each experimental replicate. The mosquitoes were then held unfed in the cage overnight ( $\sim 12$ hours) at $25^{\circ} \mathrm{C}$ until release the next day.

Mosquito release and apartment setup. Mosquitoes were transported to the apartment in an insulated cooler, and the holding cage was exposed to ambient conditions in the apartment for 10 minutes before being released. Specificcolored mosquitoes (20) were released at 10:40 AM into the center of each room with the door between the two rooms left closed (Table 1). Temperature and relative humidity within the apartment were recorded in one room using a TinyTag Gemini Data Logger (Chichester, West Sussex, UK).

Assessment of mosquito biting, movement, and mortality. The experimental rooms were left vacant for 20 minutes after mosquito release. A human landing count (performed while sitting) was then conducted to assess biting rates before initiating treatments. The count involved recording all mosquito landings on the lower right leg (exposed area was knee to toe) in which probing was initiated, before a hand was used to flush mosquitoes off the leg, over a 10-minute period. Landing counts were conducted by the same human subject for the entire experiment. The metofluthrin or coil, placed on a $0.4 \mathrm{~m}$ high box located 1.2 from the chair used for the biting assessments, was then activated. The door between the treated and neighboring room was then opened. Additional biting assessments were undertaken 1,4 , and 24 hours post-treatment at the same time each day to account for periodicity effects in mosquito behavior (Table 1). During biting assessments, the bedroom door was closed to prevent mosquitoes moving between rooms.

Mosquito movement and mortality was assessed at the completion of each treatment replicate by tallying both alive and dead mosquitoes in each room, and identifying from which room the mosquitoes were released by recording their marked color when placed under an ultraviolet light. To determine the influences of mosquito harborage sites, the location of dead mosquitoes was also recorded in relation to each item placed in the rooms for mosquito harborage.

Data analyses. Mosquito biting data, obtained through human landing counts of female Ae. aegypti, were square root $(\mathrm{X}+0.5)$ transformed to normality. A one-way analysis of variance (ANOVA) and type III error was then used to test for treatment effects at each of the count periods (pretreatment and 1,4, and 24 hours after the application of the treatment) for both the treated and untreated rooms. To identify significantly different treatments, the least significant difference (LSD) post-hoc test was applied. At the end of

TABLE 1

Schedule for assessing mosquito behavior for all treatments

\begin{tabular}{cl}
\hline Time of day & \multicolumn{1}{c}{ Activity } \\
\hline $10: 40 \mathrm{AM}$ & Mosquitoes released \\
$11: 00 \mathrm{AM}$ & Pre-treatment landing count \\
$11: 30 \mathrm{AM}$ & Treatment added to one room \\
$12: 30 \mathrm{PM}$ & 1 hour landing count \\
$3: 30 \mathrm{PM}$ & 4 hour landing count \\
$11: 30 \mathrm{AM}$ & 24 hour landing count \\
$12: 00$ noon & Collection and assessment of \\
& movement and mortality \\
\hline
\end{tabular}




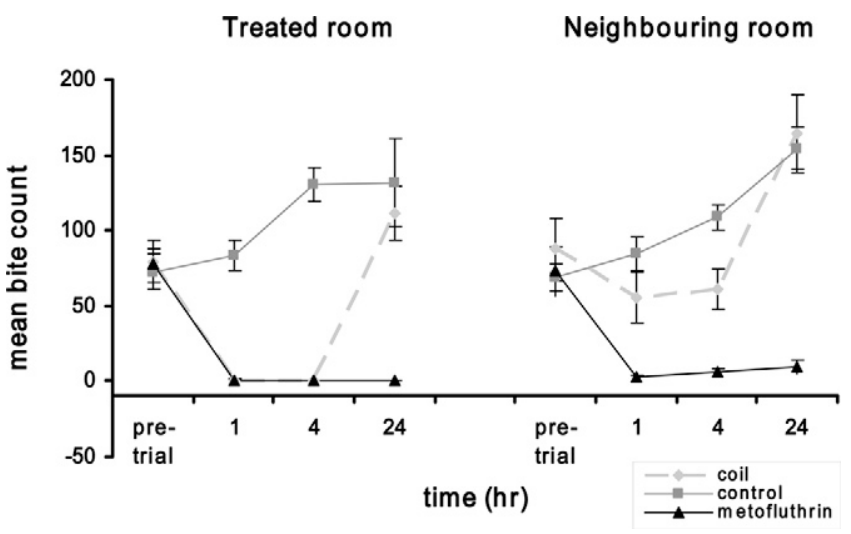

Figure 2. Mean mosquito landing counts $( \pm \mathrm{SE})$ for both the treated and neighboring rooms conducted pre-trial and 1,4 , and 24 hours post-treatment.

the 24-hour exposure period an assessment of mosquito movement was undertaken by determining the proportion of each mosquito cohort present in each room. The proportion of mosquitoes that had remained, exited, or were lost in relation to the room in which they were released (treated or untreated) was also determined. The proportion of dead mosquitoes in each room was determined for each cohort. Proportional data were arcsine square-root transformed then analyzed by ANOVA and LSD post-hoc test. All analyses were performed in SPSS (version 14.0, SPSS Inc., Chicago, IL).

\section{RESULTS}

Temperature and relative humidity. June temperatures ( mean minimum $=22.7$; mean maximum $\left.=23.8^{\circ} \mathrm{C}\right)$ and relative humidity ( mean minimum $=68.1 \%$; mean maximum $=$ $80.4 \%$ ) were higher than the temperatures (mean minimum $=$ 18.8 ; mean maximum $=22.7^{\circ} \mathrm{C}$ ) and relative humidity (mean minimum $=51.5 \%$; mean maximum $=66.6 \%$ ) recorded throughout July.

Mosquito biting: treated room. The pretreatment 10 minutes landing rate was positively correlated with temperature in the treated $(r=0.80)$ and untreated $(r=0.81)$ rooms. Metofluthrin was extremely effective at preventing biting in the treated room, reducing landing counts to zero for all metofluthrin treatments during assessments made at 1, 4, and 24 hours post-treatment (Figure 2). Similar results, although not $100 \%$ biting inhibition was recorded with the coil at the 1 and 4 hour assessments. However, at the 24 hour assessment the landing count was not significantly different (Table 2) from the control
(Figure 2). We suspect that the increase in landing rates was from mosquitoes that had recovered after the coil burned out after 8 hours.

Mosquito biting: neighboring (untreated) room. The three treatments had a significantly different impact on Ae. aegypti landing rates in the neighboring room (Table 2). Although human landing counts were not reduced to absolute zero in the neighboring room, the spillover effect of metofluthrin resulted in significantly (Table 2) fewer landings than with the control at 1, 4, and 24 hour post-treatment (Figure 2). The coil only resulted in a significant reduction in landing counts at the 4 hour assessment (Table 2), albeit less than that recorded for metofluthrin (Figure 2).

Mosquito movement. The majority of mosquitoes released in the treated and untreated rooms moved readily between rooms for all treatments, and only a small percentage of mosquitoes was not recovered ("lost") (Figure 3 ). A significantly ( $F=4.88$, $P=0.023$ ) higher proportion of mosquitoes were found in the treated room after the metofluthrin treatment than with either the coil or control treatments (Figure 4). This was a result of the knockdown of a greater number of mosquitoes entering the treatment room from the neighboring room compared with either the coil (LSD, $P=0.006$ ) or control treatment (LSD, $P=0.088$ ). Indeed, after 24 hours $58.3 \%$ of mosquitoes released into the neighboring room had exited that room and were found in the room in which the metofluthrin tower had been activated, compared with $27.5 \%$ and $40.8 \%$ for the coil and control treatments, respectively (Figure 5). There was no significant difference $(F=2.02, P=0.167)$ between treatments for the number of mosquitoes leaving the treated room (Figure 5).

Mosquito mortality. Mortality was very low for the control and coil treatments, but much higher for both cohorts in the metofluthrin treatment (Figure 3). At the household level (i.e., mortality pooled from both rooms) it resulted in a significantly $(F=112.73, P<0.001)$ greater proportion of dead mosquitoes, compared with either the coil or control (Figure 6). Mosquito collections after 24 hours revealed that $98 \%$ of mosquitoes collected in the room in which the metofluthrin tower was activated were dead. This was significantly $(F=174.01$, $P<0.001)$ higher mortality compared with negligible deaths for the other two treatments. Similar to the landing count results, metofluthrin had a spillover effect into the neighboring room causing significantly $(F=16.96, P<0.001)$ higher mortality ( $>1 / 3$ of mosquitoes collected) compared with the coil and control treatments. In the treated room $(131,85 \%)$ dead mosquitoes were found associated (on or very close to) with harborage areas (suitcase, hamper, wardrobe, etc.) with only 12 and 10, respectively, found at the window and door. In the

TABLE 2

Statistical results (one-way ANOVA and post hoc LSD) for mosquito landing counts for both the treated and neighboring rooms conducted pre-treatment application (pre-trial) and 1, 4, and 24 hours after treatment application*

\begin{tabular}{|c|c|c|c|c|c|}
\hline Room & Test & Pre-trial & 1 hour & 4 hours & 24 hours \\
\hline Treated & $\begin{array}{l}\text { ANOVA } \\
\text { LSD }\end{array}$ & $\begin{array}{l}F=0.08, P=0.924 \\
\text { Control }^{\mathrm{a}} \text { coil }^{\mathrm{a}} \\
\text { metofluthrin }^{\mathrm{a}}\end{array}$ & $\begin{array}{l}F=214.50, P<0.001 \\
\text { Control }^{\mathrm{a}} \text { coil }^{\mathrm{b}} \\
\quad \text { metofluthrin }^{\mathrm{b}}\end{array}$ & $\begin{array}{l}F=421.46, P<0.001 \\
\text { Control }^{\mathrm{a}} \text { coil }^{\mathrm{b}} \\
\text { metofluthrin }^{\mathrm{b}}\end{array}$ & $\begin{array}{l}F=29.54, P<0.001 \\
\text { Control }^{\mathrm{a}} \text { coil }^{\mathrm{a}} \\
\text { metofluthrin }^{\mathrm{b}}\end{array}$ \\
\hline Neighboring & $\begin{array}{l}\text { ANOVA } \\
\text { LSD }\end{array}$ & $\begin{array}{l}F=0.30, P=0.746 \\
\text { Control }^{\mathrm{a}} \text { coil }^{\mathrm{a}} \\
\text { metofluthrin }^{\mathrm{a}}\end{array}$ & $\begin{array}{l}F=29.49, P<0.001 \\
\text { Control }^{\mathrm{a}} \text { coil }^{\mathrm{a}} \\
\text { metofluthrin }^{\mathrm{b}}\end{array}$ & $\begin{array}{l}F=38.00, P<0.001 \\
\text { Control }^{\mathrm{a}} \text { coil }^{\mathrm{b}} \\
\quad \text { metofluthrin }^{\mathrm{c}}\end{array}$ & $\begin{array}{l}F=50.23, P<0.001 \\
\text { Control }^{\mathrm{a}} \text { coil }^{\mathrm{a}} \\
\text { metofluthrin }^{\mathrm{b}}\end{array}$ \\
\hline
\end{tabular}

* ANOVA = analysis of variance; $\mathrm{LSD}=$ least significant difference.

a,b,c Represent post hoc LSD grouping. Treatments with the same letter did not differ significantly. 


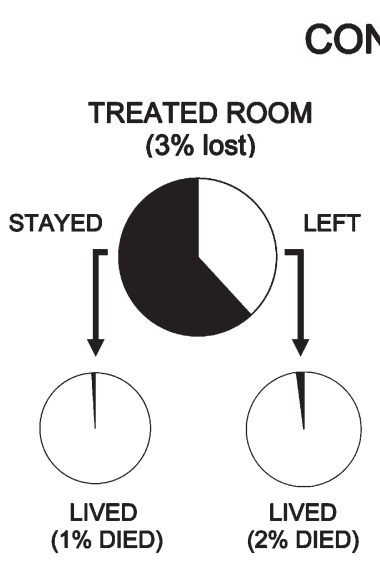

\section{CONTROL}

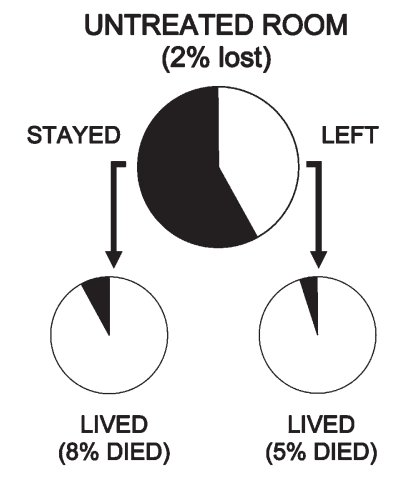

COIL
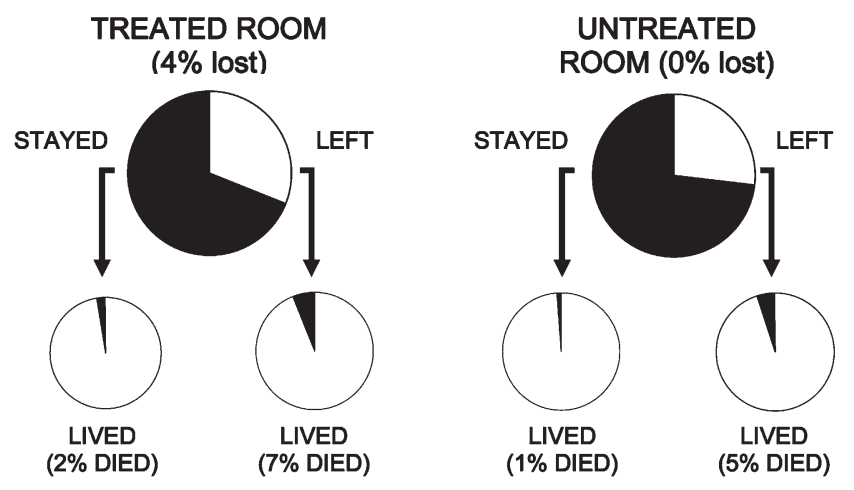

\section{METOFLUTHRIN}
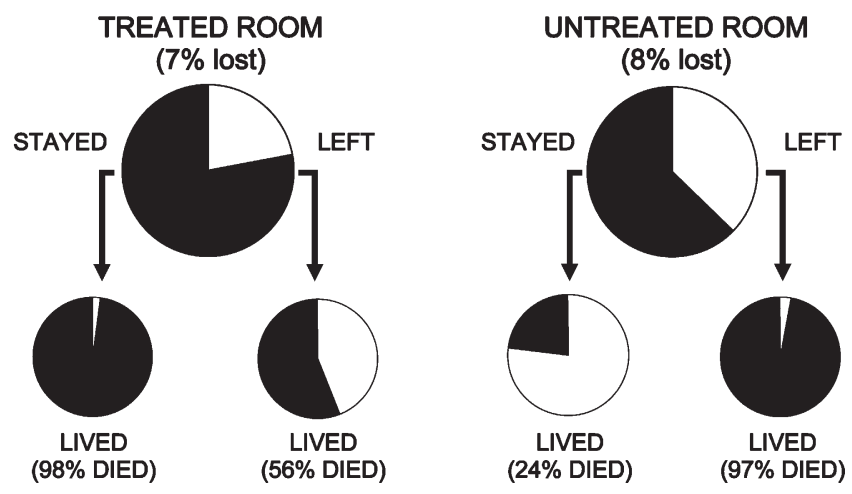

FIGURE 3. Movement (mean \pm SE) and fate of cohorts of 20 female Ae. aegypti released in treated and untreated conjoined rooms.

untreated neighboring room, 20/23 (87\%) dead mosquitoes were found in the harborage areas, with only 2 and 1 found near the window and door, respectively. These proportions were not significantly different $\left(\chi^{2}=0.85, P=0.66\right)$ between treated and untreated rooms. Not surprisingly, a high proportion of mosquitoes released in the metofluthrin-treated room died; however, the spillover of metofluthrin into the neighboring room also resulted in high mortality of mosquitoes released in that room (Figure 7). Mortality was significantly higher for metofluthrin treatment than the other treatments for mosquitoes released in the treated $(F=108.77, P<0.001)$ and untreated neighboring room $(F=47.19, P<0.001)$.

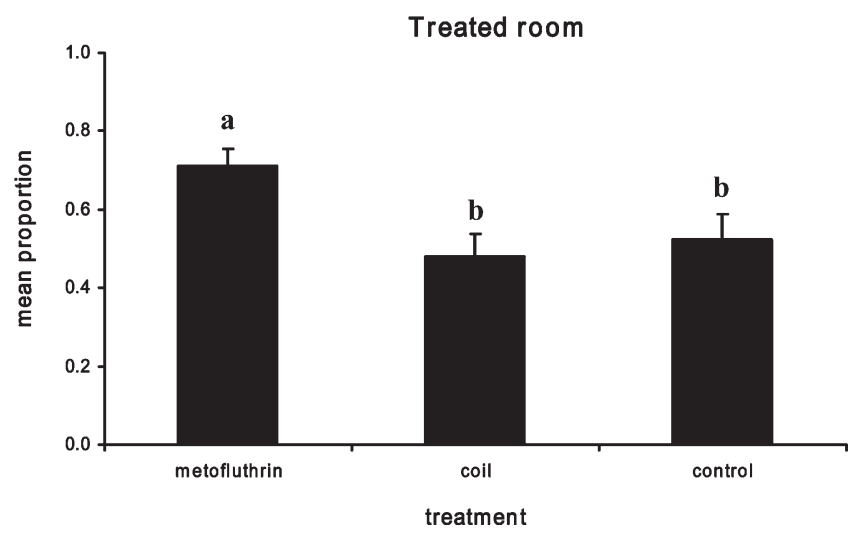

Figure 4. Mean (+SE) proportion of mosquitoes collected in the treated room for all mosquitoes collected after the 24-hour treatment exposure. Means with the same letter did not differ significantly (posthoc LSD grouping).

\section{DISCUSSION}

Throughout tropical regions of the world and Australia, dengue outbreaks are contained using mosquito control programs designed to reduce Ae. aegypti populations, thereby diminishing mosquito biting and the risk of dengue transmission. ${ }^{20}$ Although this approach may be effective in limiting dengue transmission, there remains, albeit reduced, the potential for viremic cases and their nearest neighbors to be bitten by mosquitoes remaining in the area after control measures.

The metofluthrin-impregnated tower has great potential to further improve dengue control programs, as our study has shown it to be effective in reducing mosquito biting in a typical domestic setting. Previously, in laboratory studies, Lucas and others ${ }^{9}$ found metofluthrin-impregnated paper strips reduced Ae. aegypti human landings by $91.2 \%$, and further, Lucas and others ${ }^{8}$ found metofluthrin significantly reduced human landings by Aedes spp. in the field. It is suspected that metofluthrin causes a reduction in human biting through two main modes of pyrethroid action, first as a result of its knockdown activity and second because of the disruption in orientation toward the host. ${ }^{15}$ In our study we showed that both the coil and, particularly, the metofluthrin emanator, reduced biting by Ae. aegypti while the products were active and, with the metofluthrin treatment, there was a strong spillover effect that significantly reduced biting in the adjacent untreated room.

We also found metofluthrin to be highly lethal to Ae. aegypti in the confined space of an apartment. The lethality of metofluthrin had been demonstrated by Kawada and others ${ }^{16}$ in the laboratory, where it caused $100 \%$ mortality of caged Anopheles balabacensis when exposed at a distance of 2.4 meters over a 24-hour period. In the field, however, Kawada and others ${ }^{15}$ found no significant differences in the ages, determined by parity, of Ae. aegypti collected between metofluthrin-treated and untreated houses and concluded that metofluthrin did not have a great impact on mosquito survival.

From aspirator collections in metofluthrin-treated and untreated shelters without walls ${ }^{13,14}$ and houses, ${ }^{15-17}$ Kawada and others reported that metofluthrin caused significant spatial repellency. However, in our experiments, metofluthrin did not act as either a repellent or an expellant. Had metofluthrin been operating as a repellent, mosquitoes in the neighboring room would not have entered the room in which the meto- 

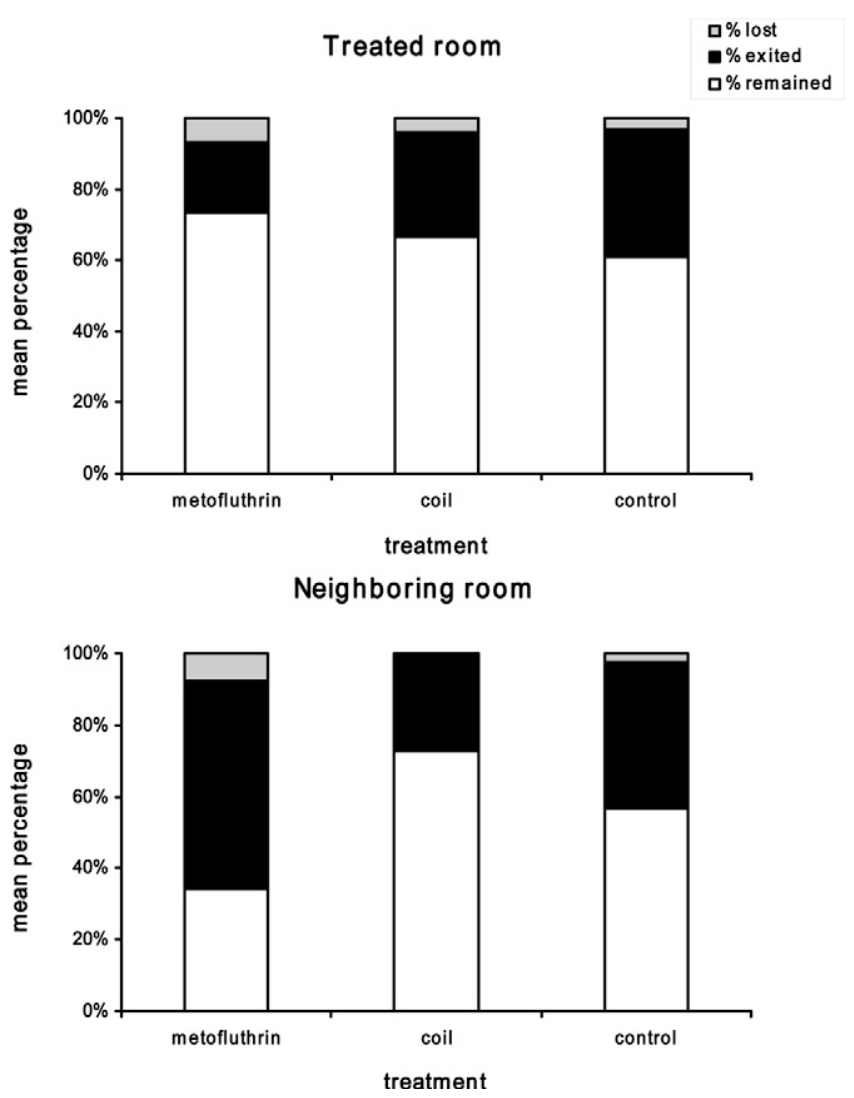

FIGURE 5. Mean percentage of mosquitoes released $(N=20)$ into the treated room and neighboring room that were lost, remained, or exited after 24-hour exposure to the metofluthrin, coil, and control treatments.

fluthrin tower had been activated; however, we did not use exit traps to measure mosquitoes exiting windows, and may have thus underestimated repellency by preventing mosquito escape via open windows. Conversely, our mosquitoes were more likely to be found, albeit dead, in the room containing the metofluthrin tower, and most dead mosquitoes were found on harborage objects in the treated room rather than at the window, which we hypothesized would be the case if they had been trying to exit.

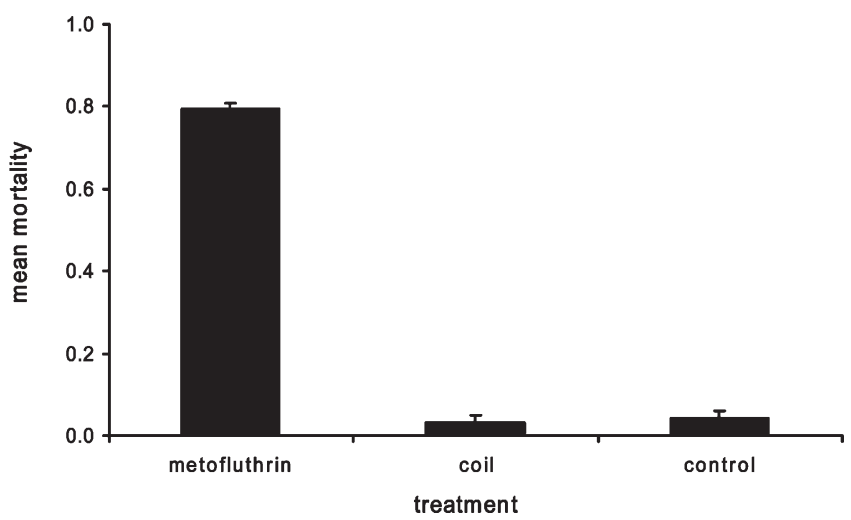

Figure 6. Mean (+SE) proportion of dead mosquitoes for the household (pooled treatment and neighboring room) after 24-hour treatment exposure. The metofluthrin treatment was significantly different from the coil and control by post-hoc LSD grouping.

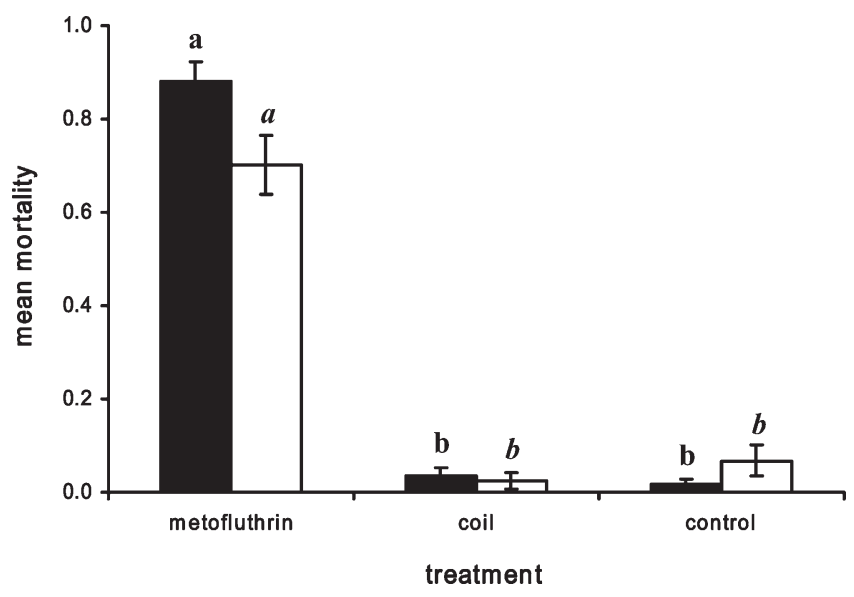

FIGURE 7. Mean cohort mortality $( \pm$ SE) for the 20 mosquitoes released in either the treated (black bar) or neighboring (white bar) room after 24-hour treatment exposure. Post-hoc LSD groupings are given for the treated and neighboring rooms (italics).

Thus, our data suggests that mosquitoes released in the neighboring room entered the treated room searching for their first blood meal, whence they became disoriented, sought harborage, and were subsequently knocked down and killed by the metofluthrin action. Indeed, disoriented mosquitoes not attempting to land or bite on an exposed leg were observed flying in the treated room during the 1 hour post-treatment landing count. Furthermore, if metofluthrin was operating as expellant in the current study, then mosquitoes released into the metofluthrin-treated room would have exited that room. However, we found no difference in the number of mosquitoes leaving the metofluthrin-treated room compared with the untreated room. Some earlier field studies using metofluthrin relied upon collection of adult mosquitoes in treated and untreated rooms using aspirators or glass vials, ${ }^{13,15}$ and thus mosquitoes that were knocked down and killed may have been overlooked. Also, disoriented mosquitoes may have flown beyond the metofluthrin-affected air, then recovered and dispersed. It is also possible that mosquitoes were attracted by metofluthrin.

Knowledge of the behavior of female Ae. aegypti exposed to metofluthrin is critical for the successful implementation of this control method. In particular, we must know whether female Ae. aegypti exposed to metofluthrin either become disorientated and are knocked down or rapidly exit the room through open windows and doors. If the latter, they would likely fly to nearby houses where they could potentially infect residents. Clearly, detailed observational studies quantifying the behavioral response of Ae. aegypti exposed to metofluthrin should be conducted under controlled field conditions to clarify this issue. Additionally, our studies were conducted at relatively cool temperatures (mean daily temperature in the low $20^{\circ} \mathrm{C}$ ). This would have decreased biting activity and vaporization of metofluthrin. Further work should be conducted to determine the relationship of temperature to efficacy and longevity of the metofluthrin emanator.

There are interesting control implications raised by the possibility that metofluthrin can kill, but not repel mosquitoes. This suggests that, when paired with humans who would act as bait, metofluthrin could be the ultimate "Lure and Kill" trap. Multiple emanators of metofluthrin could be deployed 
within a household to minimize the risk of dengue transmission to residents. However, this strategy is not without risk, as attracted mosquitoes may still bite before being disorientated by the metofluthrin. Further studies should be conducted to determine how quickly metofluthrin stops biting activity in Ae. aegypti attracted to a host. Nonetheless, area-wide deployment of metofluthrin emanators could be used to rapidly kill Ae. aegypti and interrupt virus transmission in dengue foci. However, efforts to increase the duration that metofluthrin is released from emanators (labeled for 80 hours continuous use) should be made. Nevertheless, field trials using metofluthrin over large contiguous areas are warranted. Registered metofluthrin emanators, in concert with biodegradable lethal ovitraps ${ }^{21}$ could be provided to the public as part of a "do it yourself" community participation dengue control strategy. If metofluthrin emanators were commercially available, media campaigns and websites (e.g., http://www.health.qld.gov.au/ dengue/default.asp) encouraging their use during an outbreak could be effective. Echoing this point, Morrison and oth$\mathrm{ers}^{22}$ highlighted the need to develop and test products that appeal to the consumer for the control of Ae. aegypti. Finally, our study indicates that coils provide good repellency within a room while the coil is burning. However, they do not appear to kill mosquitoes and would be of limited value in breaking dengue transmission.

Received August 6, 2008. Accepted for publication March 28, 2009.

Acknowledgments: We thank Reckitt Benckiser, particularly Michael Gibian, Graeme Smith, and Ian Thompson for providing the metofluthrin-impregnated towers and offering feedback on experimental design and results. The assistance of Petrina Johnson in locating the apartment and preparing the manuscript is gratefully acknowledged. Avril Underwood is also thanked for helping to make the apartment suitable for mosquito release and for collecting initial data on mosquito behavior when exposed to metofluthrin.

Financial support: The study was funded by the National Health and Research Medical Council (grant no. 379615) and Reckitt Benckiser Australia Pty Ltd.

Authors' addresses: Luke Rapley and Scott Ritchie, School of Public Health, Tropical Medicine and Rehabilitation Sciences, James Cook University, PO Box 6811, Cairns, QLD 4870, Australia. Richard Russell, Department of Medical Entomology, University of Sydney, Westmead Hospital, Westmead, NSW 2145, Australia. Brian Montgomery, Tropical Population Health Network, Queensland Health, PO Box 1103, Cairns, QLD 4870, Australia.

\section{REFERENCES}

1. Farrar J, Focksz D, Gubler D, Barrera R, Guzman MG, SimmonsI C, Kalayanarooj S, Lum L, McCall PJ, Lloyd L, Horstick O, DayaI-Drager R, Nathan MB, Kroeger A, 2007. Towards a global dengue research agenda. Trop Med Int Health 12: 695-699.

2. Gubler D, 1998. Resurgent vector-borne diseases as a global health problem. Emerg Infect Dis 4: 442-449.

3. Perich MJ, Kardec A, Braga IA, Portal IF, Burge R, Zeichner BC, Brogdon WA, Wirtz RA, 2003. Field evaluation of a lethal ovitrap against dengue vectors in Brazil. Med Vet Entomol 17: 205-210.

4. Sithiprasasna R, Mahapibul P, Noigamol C, Perich MJ, Zeichner BC, Burge B, Norris SLW, Jones JW, Schleich SS, Coleman RE, 2003. Field evaluation of a lethal ovitrap for the control of Aedes aegypti (Diptera: Culicidae) in Thailand. J Med Entomol 40: 455-462.
5. Scott TW, Chow E, Strickman D, Kittayapong P, Wirtz RA, Lorenz LH, Edman JD, 1993. Blood-feeding patterns of Aedes aegypti (Diptera, Culicidae) collected in a rural Thai village. $J$ Med Entomol 30: 922-927.

6. Scott TW, Clark GG, Lorenz LH, Amerasinghe PH, Reiter P, Edman JD, 1993. Detection of multiple blood feeding in Aedes aegypti (Diptera: Culicidae) during a single gonotrophic cycle using a histologic technique. J Med Entomol 30: 94-99.

7. Montgomery BL, Ritchie SA, 2002. Roof gutters: a key container for Aedes aegypti and Ochlerotatus notoscriptus (Diptera: Culicidae) in Australia. Am J Trop Med Hyg 67: 244-246.

8. Lucas JR, Shono Y, Iwasaki T, Ishiwatari T, Spero N, 2005. Field efficacy of metofluthrin-a new mosquito repellent. Lee C-Y, Robinson WH, eds. Proceedings of the Fifth International Conference of Urban Pests. Perniagaan Ph'ng, P \& Y Design Network, Malaysia.

9. Lucas JR, Shono Y, Iwasaki T, Ishiwatari T, Spero N, Benzon G, 2007. US laboratory and field trials of metofluthrin (SumiOne (R)) emanators for reducing mosquito biting outdoors. $J$ Am Mosq Control Assoc 23: 47-54.

10. Yap HH, Tan HT, Yahaya AM, Baba R, Loh PY, Chong NL, 1990. Field efficacy of mosquito coil formulations containing D-Allethrin and D-Transallethrin against indoor mosquitos especially Culex quinquefasciatus say. Southeast Asian J Trop Med Public Health 21: 558-564.

11. Liu W, Zhang J, Hashim JH, Jalaludin J, Hashim Z, Goldstein BD, 2003. Mosquito coil emissions and health implications. Environ Health Perspect 111: 1454-1460.

12. Ujihara K, Mori T, Iwasaki T, Sugano M, Shono Y, Matsuo N, 2004. Metofluthrin: a potent new synthetic pyrethroid with high vapor activity against mosquitoes. Biosci Biotechnol Biochem 68: $170-174$.

13. Kawada H, Maekawa Y, Takagi M, 2005. Field trial on the spatial repellency of metofluthrin-impregnated plastic strips for mosquitoes in shelters without walls (beruga) in Lombok, Indonesia. J Vector Ecol 30: 181-185.

14. Kawada H, Maekawa Y, Tsuda Y, Takagi M, 2004. Trial of spatial repellency of metofluthrin-impregnated paper strip against Anopheles and Culex in shelters without walls in Lombok, Indonesia. J Am Mosq Control Assoc 20: 434-437.

15. Kawada H, Iwasaki T, Loan LL, Tien TK, Mai NTN, Shono Y, Katayama Y, Takagi M, 2006. Field evaluation of spatial repellency of metofluthrin-impregnated latticework plastic strips against Aedes aegypti (L.) and analysis of environmental factors affecting its efficacy in My Tho City, Tien Giang, Vietnam. Am J Trop Med Hyg 75: 1153-1157.

16. Kawada H, Maekawa Y, Tsuda Y, Takagi M, 2004. Laboratory and field evaluation of spatial repellency with metofluthrin-impregnated paper strip against mosquitoes in Lombok Island, Indonesia. J Am Mosq Control Assoc 20: 292-298.

17. Kawada H, Yen NT, Hoa NT, Sang TM, Dan NV, Takagi M, 2005. Field evaluation of spatial repellency of metofluthrin impregnated plastic strips against mosquitoes in Hai Phong city, Vietnam. Am J Trop Med Hyg 73: 350-353.

18. Argueta TBO, Kawada H, Takagi M, 2004. Spatial repellency of metofluthrin-impregnated multilayer paper strip against Aedes albopictus under outdoor conditions, Nagasaki, Japan. Medical Entomology and Zoology 55: 211-216.

19. Russell RC, Webb CE, Williams CR, Ritchie SA, 2005. Markrelease-recapture study to measure dispersal of the mosquito Aedes aegypti in Cairns, Queensland, Australia. Med Vet Entomol 19: 451-457.

20. Ritchie S, Hanna J, Hills S, Piispanen J, McBride W, Pyke A, Spark $\mathrm{R}, 2002$. Dengue control in north Queensland, Australia: case recognition and selective indoor residual spraying. WHO Dengue Bull 26: 7-13.

21. Ritchie SA, Long SA, McCaffrey N, Key C, Lonergan G, Williams $\mathrm{CR}, 2008$. A biodegradable lethal ovitrap for control of containerbreeding Aedes. J Am Mosq Control Assoc 24: 47-53.

22. Morrison AC, Zielinski-Gutierrez E, Scott TW, Rosenberg R, 2008. Defining challenges and proposing solutions for control of the virus vector Aedes aegypti.PLoS Med 5: e68, doi:10.1371/ journal.pmed.0050068. 